\title{
Mouse models of medulloblastoma
}

\author{
Xiaochong Wu, Paul A. Northcott, Sidney Croul and Michael D. Taylor
}

\begin{abstract}
Medulloblastoma is the most common malignant pediatric brain tumor. Despite its prevalence and importance in pediatric neuro-oncology, the genes and pathways responsible for its initiation, maintenance, and progression remain poorly understood. Genetically engineered mouse models are an essential tool for uncovering the molecular and cellular basis of human diseases, including cancer, and serve a valuable role as preclinical models for testing targeted therapies. In this review, we summarize how such models have been successfully applied to the study of medulloblastoma over the past decade and what we might expect in the coming years.
\end{abstract}

Key words Genetically engineered mouse, medulloblastoma, mouse model

As the most common malignant childhood brain tumor, medulloblastoma (MB) is a developmentassociated embryonal tumor of the cerebellum, a component of the central nervous system (CNS) essential for motor coordination in vertebrates. Within two years of diagnosis, approximately $10 \%-15 \%$ of patients die of the disease ${ }^{[1]}$. Although $\sim 60 \%$ of $\mathrm{MB}$ patients can be cured with current therapeutic regimens, the majority of survivors suffer from severe long-term neurologic, endocrinologic, and cognitive sequelae ${ }^{[1]}$. Biological heterogeneity is a major property of $M B$, however, in the clinic the disease is segregated into standard risk and high risk groups. Completely resected tumors from patients older than 3 years with no leptomeningeal macroscopic dissemination at diagnosis are considered standard risk, whereas all others are designated high risk ${ }^{[2]}$. In 2007, the disease was histologically classified by the WHO into classic MB (approximately $50 \%$ of the disease) and four other subgroups including desmoplastic $M B$, anaplastic $M B$, large-cell $M B$, and $M B$ with extensive nodularity (MBEN) ${ }^{[3]}$. Enormous efforts have been devoted to classify and subgroup $\mathrm{MB}$ in order to improve prognosis and to reduce treatment-associated side effects. Recently, employing cytogenetic and molecular biomarkers, MB have been classified into distinct subgroups ${ }^{[1,2]}$. Multiple

\footnotetext{
Authors' Affiliation: Arthur and Sonia Labatt Brain Tumor Research Center, Hospital for Sick Children, Toronto, Ontario, M5G 1L7, Canada. Correspondlng Author Michael D. Taylor, Division of Neurosurgery, Program in Developmental and Stem Cell Biology, Hospital for Sick Children, Suite 1504, 555 University Avenue, Toronto, M5G 1X8, Canada. Email: mdtaylor@sickkids.ca.

doi: $10.5732 /$ cjc.011.10040
}

deregulated signaling pathways and cytogenetic aberrations have been identified in MBs; however, many questions about the pathogenesis of the disease remain to be answered.

Over the past two decades, mouse models have proven to be a very effective tool for answering questions about human cancers. Here, after a brief review of modelling human cancers in the mouse, we will discuss many of the prominent MB mouse models (categorized according to signaling pathway) published since 1997, when the first $\mathrm{Ptc}^{+/}$mouse model was generated. Upon conclusion, we will describe future perspectives on modelling human $\mathrm{MB}$ in the mouse.

\section{Modelling Human Cancers in the Mouse}

For the past two decades, conventional and advanced conditional genetically engineered mouse models (GEMMs) as well as xenografts passaged in the mouse have been an indispensable tool in basic cancer biology and translational cancer research ${ }^{[4]}$. As mammals, mice are closely related to humans at a physiological, biochemical, and genetic level. In addition, mice are less expensive to maintain than other mammals and are particularly amenable to genetic manipulation. During the last decade, enhanced knowledge of human and mouse genomes has facilitated genetic manipulation, permitting a better understanding of gene function and allowing more accurate modelling of human diseases. Ideally, a faithful recapitulation of human disease in genotype and phenotype is an essential element of mouse modelling. Four basic mouse models are briefly described below. 


\section{Transplantation/xenograft models}

After in vivo or in vitro manipulation, human or mouse tissues or cells can be transplanted into either immuno-compromised or wild-type mice subcutaneously, or orthotopically for studying gene function and screening therapeutic molecules. These models allow speedy, flexible, and relatively inexpensive manipulation of tissues or cells with multiple genetic and molecular approaches, such as gene knockout, ectopic expression of mutated genes, and RNA interference.

\section{Conventional GEMMs}

Classically, GEMMs are germline models, in which mice carry genetic modification in their germline and maintain the modification through breeding. In gain-of-function studies, pronuclear injection into a fertilized zygote is the most popular method to over-express or mis-express a gene in the mouse germline. In these models, depending on the regulatory elements chosen for expression, the transgene can be expressed either ubiquitously or in a tissue and/or time specific manner ${ }^{[5]}$. In contrast, gene targeting in mouse embryonic stem (ES) cells is employed to generate null or "knockout" mutations in order to study the loss-offunction.

\section{Conditional inducible GEMMs}

To overcome limitations associated with conventional GEMMs, such as embryonic or early postnatal lethality, conditional GEMMs have been created, in which genetic events can be tightly controlled spatially and temporally. In these systems, the site-specific bacterial Cre recombinase enzyme and its modified version Cre-ER are used to control the expression, or the "knockout" of a gene at a specific location, or at a specific time in development. Additional elements of temporal control can be gained through the use of tetracycline (Tet) inducible systems: Tet-off or Tet-on systems to conditionally over-express an oncogene or knockout a tumor suppressor ${ }^{[6,7]}$.

\section{shRNAi and transposon-based GEMMs}

Recently, short hairpin RNA interference (shRNAi) technology has been applied to mouse models in order to knockdown genes in vivo by introducing promoter-shRNA constructs into the mouse through conventional or conditional approaches ${ }^{[7,8]}$. Meanwhile, DNA transposon systems such as Sleeping Beauty (SB) have been employed to induce tumors through genetic engineering in the mouse and have been proven insightful in functional cancer genomics studies ${ }^{[9]}$.

\section{Modelling Human MB in the Mouse}

As the most common malignant pediatric brain tumor, MB has been extensively studied in pediatric neuro-oncology research. The different mouse modelling technologies described above have been applied to studies of MB biology, genetics, and preclinical research. Table 1 summarizes many of the genetically engineered MB mouse models currently published in the literature.

\begin{tabular}{|c|c|c|c|c|c|}
\hline Genotype & MB profile & MB incidence & Tumor latency (weeks) & Leptomeningeal metastasis & Reference \\
\hline $\mathrm{Ptc}^{+/-}$ & Shh/desmoplastic & $14 \%$ & $5-25$ & No & 15 \\
\hline $\mathrm{Ptc}^{+/-} \mathrm{P} 53^{-/-}$ & Shh/desmoplastic & $95 \%$ & $4-12$ & No & 18 \\
\hline $\mathrm{PtC}^{+/-} \operatorname{Ink} 4 \mathrm{C}^{-1-\text { or }+/-}$ & Shh/desmoplastic & $30 \%$ & $12-36$ & No & 19 \\
\hline $\mathrm{Ptc}^{+/-} \mathrm{Kip}^{-1-\text { or }+/-}$ & Shh/desmoplastic & $60 \%-70 \%$ & $16-18$ & No & 20 \\
\hline $\mathrm{Ptc}^{+/-} \mathrm{Hic1}^{-/-}$ & Shh/desmoplastic & $\sim 40 \%$ & by 25 & No & 22 \\
\hline Math1-Cre/Ptc ${ }^{\mathrm{C} / \mathrm{C}}$ & Shh/desmoplastic & $100 \%$ & $8-12$ & No & 23 \\
\hline Gfap-Cre/Ptc c/c & Shh/desmoplastic & $100 \%$ & $3-4$ & No & 23 \\
\hline Sufu ${ }^{+/-} / \mathrm{P} 3^{-/-}$ & Shh/desmoplastic & $58 \%$ & by 28 & No & 25 \\
\hline Hemizygous ND2-SmoA & Shh/desmoplastic & $48 \%$ & 25 & No & 26 \\
\hline Homozygous ND2-SmoA & Shh/desmoplastic & $94 \%$ & $4-8$ & Yes & 27 \\
\hline Gfap-Cre/Rb $b^{10 x p / / 10 x p} / T p 53^{-/- \text {or loxp/loxp }}$ & Shh/desmoplastic & $>84 \%$ & 12 & No & 30 \\
\hline $\mathrm{Lig}^{-/-/} \mathrm{p} 53^{-/-}$ & Shh/desmoplastic & $100 \%$ & $3-9$ & No & 32 \\
\hline Nestin-Cre/Xrcc $4^{10 \times 0 / 10 \times x} /{\mathrm{p} 53^{-/-}}^{-1}$ & Shh/desmoplastic & $87 \%$ & $12-14$ & No & 33 \\
\hline Nestin-Cre/Xrcc2 $2^{10 x p / 10 x p / p 53^{-/-}}$ & Shh/desmoplastic & $>90 \%$ & $14-16$ & No & 34 \\
\hline Nestin-Cre/Lig4 $4^{\operatorname{lox} / / 10 x p} / p^{1} 53^{-/-}$ & Shh/desmoplastic & $>90 \%$ & $14-16$ & No & 34 \\
\hline Nestin-Cre/Brca2 $2^{10 \times 0 / 10 \times x / p 53^{-/-}}$ & Shh/desmoplastic & $>90 \%$ & $14-16$ & No & 34 \\
\hline Parp1 $1^{-1-/ p 53^{-/-}}$ & Shh/desmoplastic & $49 \%$ & $8-24$ & No & 36 \\
\hline GTML & Classic or LCA & $75 \%$ & by 29 & Yes & 37 \\
\hline Blbp-Cre/Ctnnb1 $1^{+/ \operatorname{lox}(\mathrm{ex} 3)} / \mathrm{Tp} 53^{\text {fix/fix }}$ & Wnt-subgroup & $15 \%$ & $\sim 41$ & No & 39 \\
\hline
\end{tabular}


A signature feature of childhood $M B$ is its occurrence in the developing cerebellum. Development of the cerebellum begins during embryogenesis and continues after birth in both mice and humans. Two distinct germinal zones, the primary and secondary, containing stem and/or progenitor cell populations, give rise to the various cell types of the mature cerebellum ${ }^{[3]}$. The primary germinal zone, the ventricular zone (VZ), is located in the roof of the fourth ventricle, giving rise to GABAergic neurons, Purkinje cells (a specialized neuronal population of the cerebellum), and Golgi neurons. Progenitor cells of the secondary germinal zone originate in the rhombic lip and give rise to cerebellar granule neuron precursors (CGNPs) that migrate rostrally across the cerebellum to form the external granule/germinal layer (EGL). The EGL persists until postnatal day 21 (P21) in mice and into the second year of life in humans. As the cerebellum develops, CGNPs forming the EGL undergo a period of rapid and massive clonal expansion with a peak at $\mathrm{P} 5-7$ in the mouse before migrating inward, across the Purkinje cell layer, to eventually form the post-mitotic neurons of the internal granule layer (IGL). Extensive studies have shown that multiple signal transduction pathways are involved in normal cerebellar development. These pathways play a critical role in the expansion of neural precursor populations such as those found in the EGL and VZ and deregulation of these pathways are believed to contribute to MB pathogenesis. Among various developmental signaling pathways, the Wingless (WNT) and the Sonic hedgehog (Shh) pathways have been the primary area of focus for studies related to MB biology. Not surprisingly, the majority of published MB mouse models have thus been associated with these developmental signaling pathways.

\section{Shh signaling-based MB mouse models}

The morphogenic factor Shh was initially discovered in Drosophila in 1980 and since then it has been intensively studied ${ }^{[10]}$. Shh signaling plays pivotal roles in the development of multiple organs and tissues in vertebrate counterparts. As shown in Figure 1, major components in the pathway have been identified, including the negative regulators patched 1 (PTC1) and suppressor of fused (SUFU), the activating mediator smoothened (SMO), and the downstream effectors, the GLI transcription factors. There are many unanswered questions about the regulation and targets of Shh signaling. Early studies linking Shh signaling to disease identified heritable or somatic PTC mutations in patients with the basal cell nevus syndrome (BCNS, also known as Gorlin syndrome) and sporadic basal cell carcinoma $(B C C)^{[11,12]}$, as well as in sporadic MBs and other cancers ${ }^{[13,14]}$.

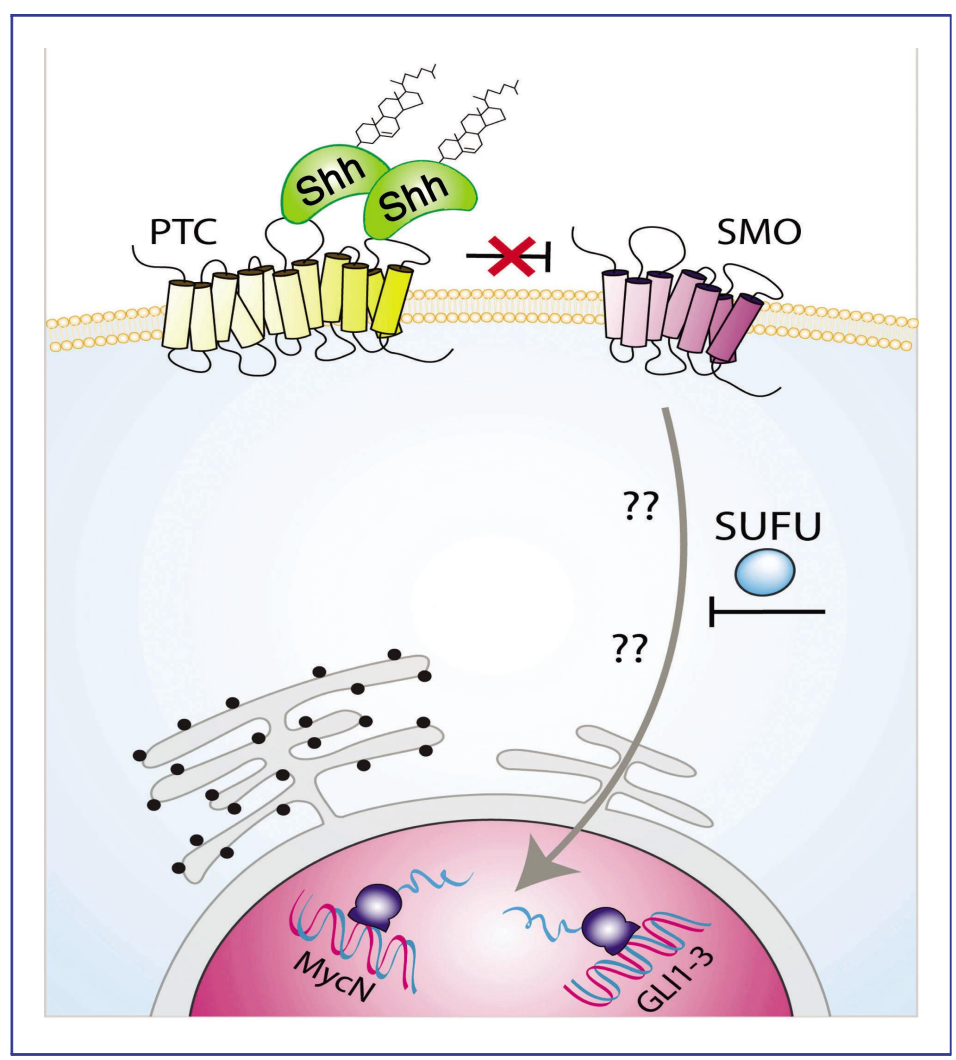

Figure 1. Major components of Sonic hedgehog (Shh) signaling pathway. In the absence of Shh, the negative regulator patched 1 (PTC1), localized at the plasma membrane, inhibits signaling through the activating mediator smoothened (SMO). In the presence of Shh ligand, Shh binds to PTC and derepresses its negative regulation on SMO, activating signaling by inducing the expression of transcription factors including $\mathrm{MycN}$ and $\mathrm{GLI}$ in the nucleus. The Shh pathway can be negatively regulated by suppressor of fused (SUFU) in the cytosol. 
The first Ptc ${ }^{+/}$mouse model To study functions of PTC in development and tumorigenesis, the first Ptc ${ }^{+-}$ mouse model was generated by homologous recombination in embryonic stem cells, in which part of Ptc exon 1 (including the putative start codon) and all of exon 2 were replaced with lac $Z$ and a neomycin resistance gene ${ }^{[15]}$. Homozygous deletion of Ptc in the mouse was embryonic lethal. In addition to an alteration in neural cell fates, $14 \%$ of $\mathrm{Ptc}^{+/}$mice (B6D2F1 background) developed $\mathrm{MB}$ as early as 5 weeks after birth, with a peak between 12 and 25 weeks, consistent with tumors seen in BCNS patients ${ }^{[15]}$. Figure 2 shows the histology of a typical MB from a $\mathrm{Ptc}^{+-}$mouse. By employing the $P^{+c^{+-}}$mouse model in 1999, Wechsler-Reya et al. ${ }^{[16]}$ were the first to show that Shh is secreted by Purkinje cells, and that Shh is responsible for regulating CGNP proliferation during normal cerebellar development. After further characterizing the first generation of the $\mathrm{Ptc}^{+-}$mouse model ${ }^{[17]}$, by crossing $P_{t C^{+-}}$mice with Tp53 deficient mice $\left(T p 53^{\prime-}\right)$, Wetmore et al. ${ }^{[18]}$ dramatically increased MB incidence to over $95 \%$ and reduced the latency to 12 weeks after birth, demonstrating that genomic instability may contribute to the pathogenesis of $\mathrm{MB}$. The high incidence of $\mathrm{MB}$ in this model may make it especially useful for preclinical studies.

Ptct-Ink4c mouse model Disruption of the RB (retinoblastoma) pathway, as a result of loss of cyclin-dependent kinase (CDK) inhibitor INK4 proteins (INK4A and INK4C) or RB itself, or over-expression of D-cyclins and their associated kinases (CDKs), is a hallmark feature of different types of human cancer ${ }^{[19]}$. To assess whether Ink4c loss could collaborate with $\mathrm{Ptc}^{+-}$ inactivation to accelerate MB formation, $\mathrm{Ptc}^{+/-}$mice were crossed with Ink4c null mice ${ }^{[19]}$. Either homozygous or hemizygous deletion of Ink4c on a $\mathrm{Ptc}^{+/-}$background greatly increased MB incidence (from 7\% to 30\%) with shortened latency although deletion of Ink $4 c$ itself was not able to induce MB formation. This result demonstrated that deregulation of the $\mathrm{Rb}$ pathway contributes to the progression of MB.

PtC ${ }^{+-}$Kip1 mouse model Recently, Kip1, another cyclin-dependent kinase inhibitor important for cerebellar development, was tested in a mouse model on a $\mathrm{Ptc}^{+-}$ background ${ }^{[20]}$. Analogous to Ink4c deletion, either homozygous or hemizygous deletion of Kip1 in $\mathrm{Ptc}^{+-}$ mice substantially increased MB incidence (from $40 \%$ to $60 \%-67 \%$ ) with a trend towards shortened latency.

Ptc ${ }^{\text {H-Hic }}{ }^{\text {t- }}$ mouse model LOH of human chromosome 17p13.3, a locus distal to Tp53, is among the most frequent genetic events in sporadic human MBs with an incidence as high as $30 \%-50 \%{ }^{[21]}$. Searching for tumor suppressor genes in this region has revealed hypermethylated in cancer 1 (HIC1), a frequent target of promoter hypermethylation and epigenetic gene silencing in $\mathrm{MB}$ and several other cancers ${ }^{[22]}$. To address the role of HIC1 in MB, Ptc ${ }^{+-}$mice were crossed with $\mathrm{Hic}^{+1-}$ mice on a $\mathrm{C} 57 \mathrm{BI} / 6$ background ${ }^{[22]}$. Although the latency of $\mathrm{MB}$ genesis in $\mathrm{Ptc}^{+-} \mathrm{Hic}^{+/-}$double heterozygous mice was similar to those in $\mathrm{Ptc}^{+-}$mice, homozygous deletion of Hic1 in Ptc ${ }^{+-}$mice had a 4-fold increase in MB incidence.

Second generation Ptc mouse model Recently the Cre-loxP system was employed to generate secondgeneration Ptc mouse models. In an attempt to identify the cell of origin of Shh signaling-associated MB, Yang et al. ${ }^{[23]}$ generated conditional Ptc-knockout mice, Math1-Cre/Ptc ${ }^{C l C}$ mice and Gfap-Cre/Ptc ${ }^{C l C}$ mice, in which homozygous deletion of Ptc was selectively carried out in granule neuron precursors (GNPs) and neuronal stem cells (NSCs), respectively. All of the conditional Ptc
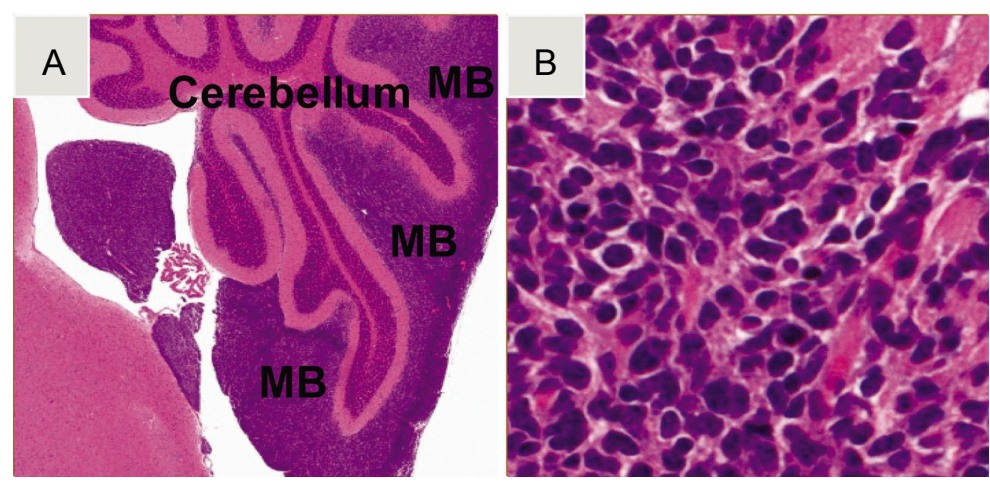

Figure 2. Histology of medulloblastoma (MB) from $\mathrm{Ptc}^{+/-}$mouse. Compared to non-tumor cells, Ptc ${ }^{+/-} \mathrm{MB}^{\mathrm{t}}$ tumor cells are stained by hematoxylin (blue) with higher intensity, showing a high ratio of nucleus to cytoplasm (stained by eosin, pink) in the poorly differentiated tumor cells. A, Hematoxylin and Eosin (H\&E) stained $M B$ at the surface of cerebellum ( $\times 10)$; B, H\&E stained MB tumor cells $(\times 40)$. 
mice, either Math1-Cre mice or Gfap-Cre mice, gave rise to $\mathrm{MB}$ incidence at the surface of cerebellum. However, the MB latency was different between the two genotypes of mice, with a latency of 3-4 weeks for Gfap-Cre/Ptc ${ }^{C / C}$ mice and 8-12 weeks for Math1-Cre/Ptc ${ }^{1 / C}$ mice. Moreover, the authors also generated Math1-Cre$E R / P t c^{C / C}$ mice to specifically delete Ptc during post-natal development by controlling Cre expression through the administration of tamoxifen.

Sufu ${ }^{+-} / T p 53^{-1-}$ mouse model In addition to Ptc, the negative regulator Sufu and the positive regulator Smo have also been employed for the establishment of Shh-dependent mouse models of MB. A subset of MB patients has been shown to carry germline and somatic SUFU mutations, implicating SUFU as a tumor suppressor ${ }^{[24]}$. To confirm the role of SUFU during development and its deregulation in MB, Lee et al. ${ }^{[25]}$ employed a gene-trap approach to generate genetically modified mice carrying a Sufu mutation. Similar to Ptc null mice, Sufu null mice died around E10. Sufu ${ }^{+-}$mice did not develop any tumors, whereas Sufut ${ }^{+1} / T p 53^{-1}$ double-mutant mice developed MBs and other types of cancer. Compared with $\mathrm{Ptc}^{+/} / \mathrm{Tp} 53^{--}$mice, in which the incidence of MBs was $\sim 93 \%$ with a latency of 2 months, $\sim 58 \%$ of $\mathrm{Sufu}^{+1} / \mathrm{Tp} 53^{--}$double-mutant mice developed MB by 4 months.

Mouse models with constitutively active Smo In order to obtain a MB mouse model with a tumor incidence higher than the first generation Ptc mouse model, Hallahan et al. ${ }^{[26]}$ generated a ND2-SmoA hemizygous mouse model, in which a constitutively active Smo was specifically expressed in GNPs under regulation of the NeuroD2 promoter. MBs were observed in $48 \%$ of the ND2-SmoA mice at median age of 25.7 weeks, indicating selectively activating Smo does increase $\mathrm{MB}$ incidence in the mouse. More recently, a ND2-SmoA homozygous mouse model ( $\mathrm{Smo} / \mathrm{Smo}$ ) was generated by the same group ${ }^{[27]}$. The homozygous model greatly increased MB incidence to $\sim 94 \%$ with a much earlier onset (4-8 weeks). More interestingly, the authors also observed leptomeningeal metastases in this mouse model that are very similar to the pattern of metastases seen in human children with MB ${ }^{[27]}$. The increased incidence, early onset, and leptomeningeal metastasis should render the $\mathbf{S}$ mo/Smo model useful for preclinical research on Shh signaling associated MBs.

\section{Tp53 mutation-based MB mouse models}

Although studies have demonstrated aberrant activation of the Shh pathway plays an essential role in a subset of MBs, mutations in this pathway, including PTC, SUFU, SMO as well as amplification of GL/1 and GLI2, are observed in only $25 \%-30 \%$ of human MB cases $^{[28]}$, indicating other genetic and/or epigenetic events are involved in MB pathogenesis. Mutations in WNT, TP53, and RB pathways as well as genes controlling histone lysine methylation have been identified in human $\mathrm{MB}^{[28,29]}$. To fully model the spectrum of human $M B$, there has been great interest in the development of Shh-independent MB mouse models. Attempts to generate a mouse model independent of Shh pathway activation have been focused on using a Tp53 null background as described below.

In fact, other than Ptc, Tp53 is the most frequently targeted gene in MB mouse modelling, in spite of the fact that Tp53 mutation (both homozygous and hemizygous deletion) alone very rarely induces $M B$ formation. Various compound mutations of Tp53 with other candidate genes, however, have been shown to be able to effectively induce $\mathrm{MB}$ in mice likely due to the genomic instability provoked by Tp53 mutation. Indeed, as mentioned earlier, Tp53 deficiency promotes MB formation in both $\mathrm{Ptc}^{+/-}$and $\mathrm{Sufu}^{+-}$backgrounds. Other than Shh signaling-associated MB mouse models, Tp53 deficiency has been shown to cooperate with the following genes in published MB mouse models.

Tp53 and $R b$ With an initial intention to study the pathogenesis of gliomas, Gfap-Cre/Rbloxp/loxp/Tp53 and Gfap-Cre/Rbloxp/loxp/Tp53 $3^{\text {loxploxp }}$ transgenic mice were generated ${ }^{[30]}$. In this study, Gfap-driven expression of Cre was detectable in newborn mice not only in the developing forebrain and spinal ganglia, but also in precursor cells located in the EGL of the developing cerebellum. Instead of gliomas, conditional inactivation of both Tp53 and Rb led to MB formation in this study. Although the cohort was small (9 mice), over $80 \%$ of the double null mice developed MBs by 3 months.

Tp53 and defective DNA damage sensor and repair genes Lig4, a nuclear ligase, is a critical component of the non-homologous end joining (NHEJ) apparatus that is required for $V(D) J$ somatic recombination and repair of DNA double strand breaks (DSBs) ${ }^{[31]}$. Lig4 homozygous mutations result in embryonic lethality in mice which however, can be rescued by Tp53 deficiency ${ }^{[31]}$. Interestingly, before the onset of pro-B lymphomas, all Lig $4^{1 / T p} 53^{-}$mice developed MBs within 9 weeks and as early as 3 weeks of age ${ }^{[32]}$. The same group went on to

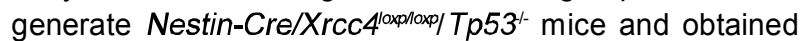
similar results as from $\mathrm{Lig}^{\mathrm{L}} / \mathrm{Tp} 53^{-}$mice with a slightly lower incidence of medulloblastoma, but no incidence of lymphoma ${ }^{[33]}$. By selectively deleting Xrcc4 (another NHEJ pathway members) in neuronal precursor cells (NPCs) on a Tp53 null background, the authors found that, along with loss of Ptc, extensive chromosomal translocations occurred in the tumor. Characterization of the induced MBs showed the tumor was similar to Ptc ${ }^{+/-}$ 
MB. More recently, to further characterize the tumor suppressor role of DNA DSB repair pathways in defined tissues, another group used the Cre-LoxP system to conditionally inactivate Lig4, Xrcc2, and Brca2 in nestin-expressing cells ${ }^{[34]}$. In combination with a Tp53 homozygous deletion, rapid development of MBs occurred in all three mouse models, indicating that both defective NHEJ and homologous recombination (HR) DNA repair pathways predispose the developing cerebellum to MB initiation. Furthermore, the specific deletion of two major tumor suppressor genes Ptc and Pten were identified in mouse models, demonstrating their important role in the suppression of $M B$ formation.

Poly-(ADP-ribose) polymerase (PARP1) is another DNA repair protein. To test the role of PARP1 in MBs, a mouse model in which Parp was homozygously deleted on a Tp53 null background was generated through crossing of Tp53 null mice with Parp null mice ${ }^{[35]}$. The Parp null mouse was generated by targeted Parp deletion in ES cells, and was healthy and fertile but impaired in response to environmental stress ${ }^{[36]}$. However, starting from 8 weeks, Parp1 homozygous deletion induced MBs in Tp53 null mice at an incidence of $\sim 49 \%$. As described above, Parp $1^{-1} / T p 53^{\prime-}$ MB showed activated Shh signaling, such as an increased expression of Gli1, and severe chromosomal aberration.

\section{Shh pathway-independent MB mouse models}

As mentioned earlier, characterization of mouse MBs generated in the context of Tp53 deficiency showed aberrant Shh signaling (particularly loss of Ptc) in the tumors, indicating they are not independent of Shh pathways, likely because in all of the models, CGNPs were the cells targeted for tumor initiation. Most recently, two models have shed light on Shh-independent MB mouse models by targeting different cell populations during CNS development.

GTML mouse model Using a tetracycline repressor-based Tet-off system, in which the tetracycline controlled transactivator (tTA) was specifically driven by the glutamate transporter 1 (G/t1) promoter, in combination with a bidirectional tetracycline response element (TRE) to express both $\mathrm{MycN}$ and luciferase, Swartling et al. ${ }^{[37]}$ recently generated a novel MB mouse model, Glt1-tTa and TRE-MycN/luciferase (Luc), which they dubbed as GTML. Although expression of N-myc in nestin-expressing cerebellar progenitors did not induce MB ${ }^{[38]}$, by 200 days, over $40 \%$ and $75 \%$ of GTML hemizygous and homozygous mice developed $M B$, respectively. Tumors were characterized as Shh-independent classic and/or LCA MBs. Remarkably, some of the mice displayed leptomeningeal metastases. These animals likely represent the first model of
non-Shh/non-Wnt MB, and should therefore prove invaluable in preclinical research. After "switching off" the MycN transgene using tetracycline, the authors were able to observe involution of the tumor, illustrating the power of tetracycline inducible models, and demonstrating the critical role of $\mathrm{MycN}$ in $\mathrm{MB}$ maintenance. We predict others will use this technology to test the role of various oncogenes not just in tumor initiation but more importantly in tumor maintenance.

Blbp-Cre/Ctnnb1+/lox(ex3)/Tp53 ${ }^{\text {hx/fix }}$-Wnt-subgroup MB mouse model Most recently, in order to validate their observations from the human disease, Gibson and colleagues generated a mouse model to recapitulate human WNT-subgroup MBs, in which, Ctnnb1 was mutated in Blbp-expressing cells via Cre-LoxP system. In combination with $T p 53^{\text {fxffx }}, 15 \%$ of the Ctnnb1 and Tp53 double mutants developed MBs (latency 290 days) with a molecular profile similar to human WNT-subgroup MBs ${ }^{[39]}$. The authors used this model to great effect to identify a candidate cell of origin for Wnt MB that differs from the cell of origin for Shh MB.

\section{Non-GEM MB mouse models}

Similar to numerous other types of cancer, both ectopic flank and orthotopic intracranial xenografts into immuno-compromised mice, such as Nu/Foxn1/Nu and NOD/SCID, have been widely used in $\mathrm{MB}$ research, especially for validation studies and preclinical research ${ }^{[40-43]}$. The extent to which human xenograft models of MB experience genetic or epigenetic "drift" overtime that causes them to lose similarity to the freshly resected tumor is not currently known.

To directly test whether the activation of the Shh pathway can lead to tumor formation, Weiner et al. ${ }^{[44]}$ created a retroviral mouse model by injecting a Shh-expressing retrovirus into the embryonic mouse cerebellum using in utero ultrasound backscatter microscopy (UBM) image guidance. MB was observed in about $76 \%$ of the experimental mice at P14 and P21. Although this model can be technically demanding, further optimization might render it useful for preclinical drug screening. Similarly, the RCAS/tv-a system is a mouse model that integrates orthotopic cell transplantation into a transgenic mouse. Dan Fult's group has been using RCAS/tv-a for simultaneously targeting multiple transgenes including $M y c N, M y c C$, lgf2, Akt, Gli1 into nestin-expressing cell populations to induce/modulate $\mathrm{MB}$ in neural progenitors of the cerebella of newborn mice ${ }^{[38,45,46]}$. In this system, RCAS, the replication-competent avian leukosis virus spliceacceptor subgroup-A (ALV-A) vectors that carry the transgenes, are transfected in vitro and the virus-producing cells are then injected into a transgenic 
mouse line that produces TV-A (the receptor for ALV-A) under a specific promoter. TV-A-mediated viral infection therefore leads to RCAS provirus integration into the specific host cell genome where the transgene is expressed. This is an excellent tool for rapidly testing genes in vivo, with the major limitation of the system being the size of the insert carried by the TV-A virus.

\section{Summary and Future Perspectives}

In summary, the majority of published MB GEM and related models are associated with the Shh signaling pathway using either a Ptc or a Tp53 mutant background. These models are based on observations from the human disease. The genetic, epigenetic and pathologic heterogeneity of MB strongly suggests other MB driver genes and pathways responsible for the initiation, maintenance and metastasis of MBs remain to be uncovered. Recently, SB transposon-based mouse models have been reported for functional cancer gene

\section{References}

[1] Pfister S, Remke M, Benner A, et al. Outcome prediction in pediatric medulloblastoma based on DNA copy-number aberrations of chromosomes $6 q$ and $17 q$ and the MYC and MYCN loci [J]. J Clin Oncol, 2009,27(10):1627-1636.

[2] Northcott PA, Korshunov A, Witt $\mathrm{H}$, et al. Medulloblastoma comprises four distinct molecular variants [J]. J Clin Oncl, 2011,29(11):1408-1414

[3] Gilbertson RJ, Ellison DW. The origins of medulloblastoma subtypes [J]. Annual Rev Pathol, 2008,3:341-365

[4] Heyer J, Kwong LN, Lowe SW, et al. Non-germline genetically engineered mouse models for translational cancer research [J] Nat Rev Cancer, 2010,10(7):470-480

[5] Ittner LM, Götz J. Pronuclear injection for the production of transgenic mice [J].Nat Protoc, 2007,2(5):1206-1215.

[6] Friedel $\mathrm{RH}$, Wurst $\mathrm{W}$, Wefers $\mathrm{B}$, et al. Transgenic mouse methods and protocols [J]. Methods Mol Biol, 2011,693:205231.

[7] Walrath JC, Hawes JJ, Van Dyke T, et al. Genetically engineered mouse models in cancer research [J]. Adv Cancer Res, 2010, 106:113-164

[8] Dickins RA, Mcjunkin K, Hernando E, et al. Tissue-specific and reversible RNA interference in transgenic mice [J]. Nat Genet, 2007,39(7):914-921

[9] Dupuy AJ. Transposon-based screens for cancer gene discovery in mouse models [J]. Semin Cancer Biol, 2010,20 (4):1-8.

[10] Jiang $\mathrm{J}$, Hui CC. Hedgehog signaling in development and cancer [J]. Dev Cell, 2008,15(6):801-812.

[11] Johnson RL, Rothman $A L$, Xie J, et al. Human homolog of patched, a candidate gene for the basal cell nevus syndrome [J]. Science, 1996,272(5268):1668-1671.

[12] Hahn H, Wicking C, Zaphiropoulous PG, et al. Mutations of the human homolog of Drosophila patched in the nevoid basal cell carcinoma syndrome [J]. Cell, 1996,85(6):841-851.

[13] Raffel C, Jenkins RB, Frederick L, et al. Sporadic discovery ${ }^{[47,48]}$

Metastasis and recurrence of $\mathrm{MB}$ is the main cause of death in patients. Currently MB patients either with or without metastasis receive similar treatments due to scarce knowledge about the biology and molecular genetics of metastasis. Among the published models, metastasis was observed only in two of them (Smo/Smo and GTML), suggesting the necessity of developing additional mouse models that can mimic both the primary tumor and its metastases.

Current developments in genomics, particularly deep sequencing technologies, promise to deliver large amounts of genetic and epigenetic data from the various subsets of human $\mathrm{MB}$ in the very near future. Determining which events are driver mutations and among those, which are required for tumor maintenance and worth targeting in the clinic, will require sophisticated mouse models to be developed and tested in the near future.

Received: 2011-02-09; revised: 2011-03-01; accepted: 2011-03-02. medulloblastomas contain PTCH mutations [J]. Cancer Res, 1997,57(21):842-845

[14] Wolter M, Reifenberger J, Sommer C, et al. Mutations in the human homologue of the Drosophila segment polarity gene patched $(\mathrm{PTCH})$ in sporadic basal cell carcinomas of the skin and primitive neuroectodermal tumors of the central nervous system [J]. Cancer Res, 1997,57(13):2581-2585

[15] Goodrich LV. Altered neural cell fates and medulloblastoma in mouse patched mutants [J]. Science, 1997,277(5329):11091113.

[16] Wechsler-Reya RJ, Scott MP. Control of neuronal precursor proliferation in the cerebellum by Sonic Hedgehog [J]. Neuron, 1999,22(1): 103-114.

[17] Wetmore C, Eberhart DE, Curran T. The normal patched allele is expressed in medulloblastomas from mice with heterozygous germ-line mutation of patched [J]. Cancer Res, 2000,60 (8): 2239-2246.

[18] Wetmore C, Eberhart DE, Curran T. Loss of p53 but not ARF accelerates medulloblastoma in mice heterozygous [J]. Cancer Res, 2001,61(2):513-516.

[19] Uziel T, Zindy F, Xie S, et al. collaborate independently with Patched to suppress medulloblastoma formation [J]. Genes Dev, 2005, 19(22):2656-2667.

[20] Ayrault O, Zindy F, Rehg J, et al. two tumor suppressors, p27Kip1 and pateched-1, collaborate to prevent medulloblastoma [J]. Mol Cancer Res, 2009,7(1):33-40.

[21] Rood BR, Zhang H, Weitman DM, et al. Hypermethylation of HIC-1 and 17p allelic loss in medulloblastoma [J]. Cancer Res, 2002,62(13):3794-3797

[22] Briggs KJ, Corcoran-Schwartz IM, Zhang W, et al. Cooperation between the Hic1 and Ptch1 tumor suppressors in medulloblastoma [J]. Genes Dev, 2008,22(6):770-785.

[23] Yang ZJ, Ellis T, Markant SL, et al. Medulloblastoma can be initiated by deletion of patched in lineage-restricted progenitors or stem cells [J].Cancer Cell, 2008,14(2):135-145. 
[24] Taylor MD, Liu L, Raffel C, et al. Mutations in SUFU predispose to medulloblastoma [J]. Nature Genet, 2002,31(3): 306-310.

[25] Lee Y, Kawagoe R, Sasai K, et al. Loss of suppressor-of-fused function promotes tumorigenesis [J]. Oncogene, 2007,26(44): 6442-6447.

[26] Hallahan AR, Pritchard JI, Hansen S, et al. The SmoA1 mouse model reveals that notch signaling is critical for the growth and survival of sonic hedgehog-induced medulloblastomas [J]. Cancer Res, 2004,64(21):7794-7800.

[27] Hatton BA, Villavicencio EH, Tsuchiya KD, et al. The Smo/Smo model: hedgehog-induced medulloblastoma with $90 \%$ incidence and leptomeningeal spread [J]. Cancer Res, 2008,68 (6): 1768-1776.

[28] Dubuc AM, Northcott PA, Mack S, et al. The genetics of pediatric brain tumors [J]. Curr Neurol Neurosci Rep, 2010,10 (3): 215-223

[29] Northcott PA, Nakahara Y, Wu X, et al. Multiple recurrent genetic events converge on control of histone lysine methylation in medulloblastoma [J]. Nature Genet, 2009,41(4): 465-472.

[30] Marino S, Vooijs M, van Der Gulden $\mathrm{H}$, et al. Induction of medulloblastomas in p53-null mutant mice by somatic inactivation of $\mathrm{Rb}$ in the external granular layer cells of the cerebellum [J]. Genes Devlop, 2000,14(8):994-1004.

[31] Frank KM, Sharpless NE, Gao $Y$, et al. DNA ligase IV deficiency in mice leads to defective neurogenesis and embryonic lethality via the p53 pathway [J]. Mol Cell, 2000,5 (6):993-1002.

[32] Lee $\mathrm{Y}$, McKinnon PJ. DNA ligase IV suppresses medulloblastoma formation [J]. Cancer Res, 2002,62(22):63956399.

[33] Yan CT, Kaushal D, Murphy M, et al. XRCC4 suppresses medulloblastomas with recurrent translocations in p53-deficient mice [J]. Proc Natl Acad Sci U S A, 2006,103(19):7378-7383.

[34] Frappart PO, Lee $Y$, Russell HR, et al. Recurrent genomic alterations characterize medulloblastoma arising from DNA double-strand break repair deficiency [J]. Proc Natl Acad Sci U S A, 2009, 106(6):1880-1885.

[35] Tong WM, Ohgaki $\mathrm{H}$, Huang $\mathrm{H}$, et al. Null mutation of DNA strand break-binding molecule poly (ADP-ribose) polymerase causes medulloblastomas in p53(-/-) mice [J]. Am J Pathol, 2003, 162(1):343-352.

[36] Wang ZQ, Auer B, Stingl L, et al. Mice lacking ADPRT and poly(ADP-ribosyl)ation develop normally but are susceptible to skin disease [J]. Genes Dev, 1995,9(5):509-520.

[37] Swartling FJ, Grimmer MR, Hackett CS, et al. Pleiotropic role for MYCN in medulloblastoma [J]. Genes Dev, 2010,24(10): 1059-1072.

[38 Browd SR, Kenney AM, Gottfried ON, et al. N-myc can substitute for insulin-like growth factor signaling in a mouse model of sonic hedgehog-induced medulloblastoma [J] Cancer Res, 2006,66(5):2666-2672.

[39] Gibson P, Tong Y, Robinson G, et al. Subtypes of medulloblastoma have distinct developmental origins [J] Nature, 2010,468(7327):1095-1099.

[40] Leggas M, Stewart CF, Woo MH, et al. Relation between Irofulven (MGl-114) systemic exposure and tumor response in human solid tumor xenografts [J]. Clin Cancer Res, 2002,8(9): 3000-3007.

[41] Kongkham PN, Northcott PA, Ra YS, et al. An epigenetic genome-wide screen identifies SPINT2 as a novel tumor suppressor gene in pediatric medulloblastoma [J]. Cancer Res, 2008,68(23):9945-9953.

[42] Ward RJ, Lee L, Graham K, et al. Multipotent CD15+ cancer stem cells in patched-1-deficient mouse medulloblastoma [J] Cancer Res, 2009,69(11):4682-4690.

[43] Kongkham PN, Northcott PA, Croul SE, et al. The SFRP family of WNT inhibitors function as novel tumor suppressor genes epigenetically silenced in medulloblastoma [J]. Oncogene, 2010,29(20):3017-3024.

[44] Weiner HL, Bakst R, Hurlbert MS, et al. Induction of medulloblastomas in mice by sonic hedgehog, independent of Gli1 [J]. Cancer Res, 2002,62(22):6385-6389.

[45] Rao G, Pedone CA, Coffin CM, et al. C-Myc enhances sonic hedgehog-induced medulloblastoma formation from nestinexpressing neural progenitors in mice [J]. Neoplasia, 2003,5 (3): 198-204.

[46] Rao G, Pedone CA, Del Valle L, et al. Sonic hedgehog and insulin-like growth factor signaling synergize to induce medulloblastoma formation from nestin-expressing neural progenitors in mice [J]. Oncogene, 2004,23(36):6156-6162.

[47] Collier LS, Carlson CM, Ravimohan S, et al. Cancer gene discovery in solid tumors using transposon-based somatic mutagenesis in the mouse [J]. Nature, 2005,436(7048):272276.

[48] Dupuy AJ, Akagi K, Largaespada DA, et al. Mammalian mutagenesis using a highly mobile somatic Sleeping Beauty transposon system [J]. Nature, 2005,436(7048):221-226. 